\title{
STRESS AND COMMUNICATION AS QUALITY INDICATORS OF A WORKING ENVIRONMENT IN LOGISTICS COMPANIES: A CASE STUDY IN THE LOGISTICS SECTOR IN SERBIA
}

\author{
Milan Andrejić ${ }^{1}$, Vukašin Pajić ${ }^{2}$, Milorad Kilibarda ${ }^{3}$ \\ ${ }^{1,2,3}$ University of Belgrade, Faculty of Transport and Traffic Engineering, Vojvode Stepe 305, 11000 \\ Belgrade, Serbia
}

Received 15 April 2020; accepted 12 June 2020

\begin{abstract}
Recently, more attention has been paid to the quality of the working environment, both in literature and in practice. The main reason for this is the increased fluctuation of workers, where the inadequate quality of the working environment is one of the motives for changing the job. Stress and communication are the two basic indicators of the quality of the working environment. For this reason, the aim of this paper is to determine the impact of stress and communication on the quality of the working environment in the logistics sector of Serbia. The impact of company size, primary business activity and employee position on stress sources, stress management and frequency of communication problems was examined. The research hypotheses were defined, which were tested on the basis of data obtained from a web survey that included 101 respondents from different industrial sectors in the Serbian market.
\end{abstract}

Keywords: stress, communication, working environment, logistics.

\section{Introduction}

In recent years, more and more attention has been paid to the health, safety and working conditions of employees. One of the main reasons for this is the raise of awareness of companies and understanding the importance of caring for their employees. One of the indicators which shows that attention is paid to the health and safety of the workers is reflected in the implementation of one of the standards dealing with this issue, such as OHSAS 18001 and ISO 45001. Another reason for the increased attention is the lack of workers and the significant fluctuation of workers who change jobs if the quality of the working environment is not at a satisfactory level. This is primarily due to the stressful and dynamic work in the logistics sector. The results of the research (Kilibarda et al., 2019) show that one of the motives for changing jobs in the logistics sector of Serbia is a hard and stressful job. The fact that more attention is being paid today to the quality of the working environment is proven by other researches (Hee et al., 2019; Ismail et al., 2015; Jacobs et al., 2015; Ližbetinova, 2015; Sadlowska-Wrzesinska and MoscickaTeske, 2016).

When it comes to stress, two types of stress need to be distinguished, acute and chronic. The effects of acute stress are reflected in problems such as headache, sleep

${ }^{1}$ Corresponding author: m.andrejic@sf.bg.ac.rs 
disturbance, short temper and problems with concentration. On the other hand, chronic (long-term) stress results in anxiety, increased blood pressure, impaired immunity and insomnia. In addition to this division, there is another that divides stress into positive (eustress), which is short-termed, motivating, and helps being more efficient and successful; and negative (distress) that causes anxiety, impedes efficiency and effectiveness, and can cause mental and physical problems. For this reason, the aim of this paper is to analyze the sources of negative stress and its impact on the quality of the working environment. Then it comes to the sources of stress, then they can be divided into internal (depending on the person) and external (depending on the environment). The research (SadlowskaWrzesinska and Moscicka-Teske, 2016) shows that the exposure of employees to the stress in the transport sector caused:

- They used sick leave often and longer remained absent from work;

- Have poorer health compared to people of similar age and the same gender;

- Were less committed to work;

- Were willing to change their job;

- Satisfaction level was significantly lower;

- Ability to work was rated worse.

In addition to stress, communication is an important indicator of the quality of the working environment. This primarily refers to internal communication, i.e. communication within one company. Namely, numerous researches show that if this type of communication is bad, it directly affects the satisfaction of the employees and therefore their performance. In addition to the effect on employees, impaired communication affects the company itself. For this reason, it is crucial for companies to understand the importance of good and open communication and to dedicate themselves to developing their employees' soft skills and organizing communication training. Apart from internal, external communication is also significant, which most often occurs between company representatives and their suppliers/clients. This form of communication can have a significant impact on the reputation of the company and certainly depends on the communication skills of the individual representing the company. Open communication in logistics can be crucial to the quality of the provided service and to the efficiency of the company. For this reason, as in the case of stress in this paper, this indicator was considered in assessing the quality of the working environment.

The paper is organized as follows. After the introduction, a problem description as well as review of the literature is presented, which is the basis for the development of hypotheses. A description of the methodology is presented in chapter three. Collected data and the results of hypothesis testing are then presented in the next chapter. At the very end of the paper, concluding remarks and directions for future research are outlined.

\section{Problem Description and Hypothesis Development}

Workplace stress is a significant problem for both employees and companies. Employee stress can create negative effects on both health and work efficiency. In addition, work stress also increases workforce fluctuation, lack of motivation and burnout (Ismail et al., 2015). On the other hand, it directly affects the company itself. Communication is one of the key areas of management that can significantly affect the effectiveness 
of an organization (Jacobs et al., 2015). For this reason, internal communication represents the basis for the success of a company, where it is of great importance that companies understand the importance of communication with employees and to observe communication as a key success factor (Jacobs et al., 2015).

One of the main sources of stress in companies is communication, most often internal, and for this reason, the aim of this paper is to determine the impact of stress and communication on the quality of the working environment in logistics companies. In the following part of the paper, a problem description, as well as a literature review dealing with this topic, is provided. In addition, the hypotheses were defined and then tested.

\subsection{Stress and Sources of Stress}

Workplace stress can be defined as a change in an employee's physical and/or mental state due to an appraised challenge or threat to one's relationship with his or her employer (Colligan and Higgins, 2006). The problems that can be caused by stress can be observed from two aspects, that of the user and the company. The first one is the employee aspect where stress affects health, quality of life, work, confidence and personal development. On the other hand, problems that can be reflected in the increase in employee turnover, decrease in quality of work, a decrease in job satisfaction and poor communication, can create a bad working environment. Stress can negatively affect both the mental and physical condition of the employee. For this reason, it is necessary to identify the causes of stress and then act on them in order to reduce or eliminate stress exposure. Cooper and Marshall
(1976) divided the main causes of stress into the following groups: intrinsic to job, role in organization, career development, relationships at work and organizational structure and climate, table 1 . Intrinsic to job stress sources are related to the working conditions that are specific to each job. These causes are mainly associated with stress resulting from work overload, time pressures and lack of employee autonomy. Work overload can be defined as too much work to do or having work that is perceived as too difficult (Cooper and Marshall, 1976). On the other hand, the problem of work overload can also arise due to insufficient knowledge of the employee. Another source of stress within this group is one of the most prevalent in practice today, and it is related to time pressure. Time pressure can be defined as a perception of insufficient time to complete a task and an awareness of the potential negative consequences of missing a deadline (Thomas et al., 2011). Due to the high volume of work that every employee encounters on a daily basis, this source of stress is inevitable and it is up to companies to better organize their work or organize additional training for employees to learn how to cope with stress. The last source of stress from this group is related to the lack of employee autonomy, i.e. the lack of decisionmaking possibilities, which is particularly pronounced in larger companies.

The second group of stress sources is related to the role in organization, where the most common causes are role conflict, external conflicts and responsibility for others. Role conflict as stress occurs in a situation where conflicting goals arise between different positions and/or sectors within the company. Namely, it is often the case that different positions and/or sectors have differently set goals and priorities which 
can lead to a conflict. These causes can also be called internal causes. In addition to internal ones, there are external conflicts that arise from conflicting goals of the company and its suppliers and/or partner companies. This is especially pronounced in situations where companies outsource logistics services from logistics providers, where they observe logistics and logistics activities as cost sources, while on the other hand, logistics providers tend to constantly reduce costs. The last source of stress within this group relates to the stress caused by responsibility for other employees. This type of stress is mainly faced by employees in higher hierarchical positions in charge of managing a certain sector. In addition to the pressure that higher position employees suffer from superiors regarding the deadlines for completing certain tasks, there is also pressure associated with a responsibility for employee's health. This is particularly pronounced in the logistics sector, where warehouse and transport managers must take into account the health and safety of employees and take all necessary measures to protect workers in the workplace.

The next group of stress sources is related to career development. All employees of one company are exposed to these sources of stress. The most common sources of stress in this group are: promotion, job security and opportunity (Cooper and Marshall, 1976). Employees who lack rewards (in the form of advancement) despite the effort they put into doing their job are particularly exposed to these sources of stress. In addition to promotion, job security is also one of the causes of stress in this group. Namely, in private companies, the fluctuation of the workforce is more pronounced in relation to the state sector, and the stress caused by the issue of job security is more pronounced among the employees in the private sector. A review of research as well as the results of the authors' research (Yaslioglu et al., 2013), showed that there is a negative correlation between stress caused by job insecurity and employee satisfaction, i.e. job insecurity has led to reduced job satisfaction. When changing jobs, the possibility of new employment is very important, where the lack of these opportunities leads to additional stress.

The fourth group of stress sources is related to relationships at work. The sources of stress in this group are: poor relationships between colleagues (Colligan and Higgins, 2006), low trust, low supportiveness and low interest in trying to manage problems (Cooper and Marshall, 1976). This group is important for both employees and companies. Namely, poor communication between employees can result in poor performance for both employees and the entire company. Also, poor communication can lead to the creation of an unfavorable working environment, from which additional sources of stress may arise. Problems with low trust and low interest in trying to manage problems can significantly affect a company's performance and compromise its effectiveness.

The last group of stress sources is related to organizational structure and climate. The sources of stress associated with this group are: communication patterns, rules and politics of the company and working environment (Colligan and Higgins, 2006). Company policies and rules can cause stress for employees when employees' opinions do not agree with company policies and/or rules. On the other hand, stress caused by the working environment can be linked to sources from the previous group such as low trust and low interest in trying to manage problems. 
Table 1

Stress Sources

\begin{tabular}{|c|c|}
\hline Stress Group & Stress Factor \\
\hline \multirow{3}{*}{ Intrinsic to job } & Work overload \\
\hline & Time pressure \\
\hline & Lack of autonomy \\
\hline \multirow{3}{*}{ Role in organization } & Role conflict \\
\hline & External conflict \\
\hline & Responsibility for others \\
\hline \multirow{3}{*}{ Career development } & Promotion \\
\hline & Job security \\
\hline & Opportunity \\
\hline \multirow{4}{*}{ Relationships at work } & Poor relationships between colleagues \\
\hline & Low trust \\
\hline & Low supportiveness \\
\hline & Low interest in trying to manage problems \\
\hline \multirow{3}{*}{ Organizational structure and climate } & Communication patterns \\
\hline & Rules and politics of the company \\
\hline & Working environment \\
\hline
\end{tabular}

When it comes to stress in logistics, then it is possible to observe and analyze stress in specific sectors of logistics. Namely, in the procurement sector, employees often face the stress associated with raw material procurement, planning, inventory monitoring, negotiations and costs of procurement. On the other hand, employees in the distribution sector face the stress associated with defining a distribution plan, delivering the right goods at the right time and place, respecting the delivery time, problems with a delivery, complaints, etc. One of the main logistics sectors in which increased stress occurs for both workers and managers is the warehouse. When it comes to stress in the warehouse, then it is possible to distinguish two types of stress, mental and physical stress. Mental stress occurs due to the increased volume of work (more picking orders) as well as due to the short deadlines provided for the preparation of goods. Apart from these main sources, the problem of internal communication between employees can also be a source of stress. Fear of possible injury when working in a warehouse is also a stress that belongs to this group. Workrelated errors, as well as penalties resulting from these errors, are also sources of stress for warehouse employees. In addition to the psychic, the workers in the warehouse are, as noted, also exposed to the physical stress resulting from the lifting of the load during the process of preparing the goods. In order to reduce the degree of exposure to this type of stress, many companies are using different types of mechanization to facilitate their work. Besides the stress caused by lifting loads, work in shifts (especially at night) causes physical stress. Another major logistics sector where increased stress occurs is transport. Based on the results (SadlowskaWrzesinska and Moscicka-Teske, 2016), it can be concluded that the most common sources of stress in this sector are time pressure and work overload (which was confirmed by $49,4 \%$ of the respondents). In addition, the stress associated with job loss has also recently been reported as a significant source $(24,9 \%$ of respondents). The results 
of the research (Sadlowska-Wrzesinska and Moscicka-Teske, 2016) show that the most significant sources of stress were the risk of job loss ( $95,8 \%$ of respondents), the risk of loss of health and/or life $(92,8 \%$ of respondents), damage/loss penalties ( $92 \%$ of respondents), filling in multiple documents (91,2\% of respondents) and rules for the granting bonuses and salaries are ambiguous (89,4\% of respondents). Strict requirements concerning the transport time stood out as another source of stress in $89,4 \%$ of the respondents.

\subsection{Communication}

Communication can be defined as the process of transmitting information and common understanding from one person to another (Adu-Oppong and AgyinBirikorang, 2014). Communication is very important in order to build and maintain good relationships with other colleagues as well as a good working environment. The importance of good communication is also reflected in the fact that, based on the information transferred on this occasion, numerous decisions can be made as well as managing different processes (Ližbetinova, 2015). As previously mentioned, poor communication between employees can have many consequences. How important communication is can best be seen based on the benefits good communication brings, such as: creates job satisfaction, lesser conflicts, increases productivity, the formation of relationships and proper utilization of resources (Adu-Oppong and Agyin-Birikorang, 2014). Companies that encourage better communication between employees at different hierarchical levels encounter less fluctuation of workers since they create satisfied workers. In addition, better and open communication also reduces potential conflicts, both internal and external. By creating satisfied workers, companies directly influence the increase in their productivity. Another benefit of open communication is the creation of better relationships between superiors and employees and between employees at the same hierarchical level. Poor communication can cause many problems such as downtime and poor utilization of available resources. Based on this, it can be concluded that one more benefit of good communication is a better utilization of resources, which directly increases the efficiency of the process.

In order for the company-level communication to be at an appropriate level, in addition to the training which companies can organize for their employees, it is also necessary for the company to choose the right communication model that can be: horizontal communication, vertical downwards communication, vertical upward communication and diagonal communication (Ližbetinova, 2015). In horizontal communication, information transfer takes place at the same hierarchical level (between employees in the same sector). The advantage of this type of communication is reflected in the higher quality of the information exchanged as it is shared between employees at the same hierarchical level, enhancing employee involvement and reducing conflict and misunderstanding (Hee et al., 2019). Horizontal communication can be further divided according to hierarchical levels into: top level management, middle management, management of the first line and executive level personnel (Ližbetinova, 2015). Vertical downwards communication is a form of communication where information is transmitted from hierarchically higher levels to hierarchically lower levels. On the other hand, vertical upward communication 
involves the transfer of information from hierarchically lower levels to hierarchically higher levels, all with the aim of defining plans, goals and strategies. Diagonal communication involves the existence of teams that are tasked to transfer information when solving business problems. Based on the research (Hee et al., 2019), it was concluded that downward and horizontal communication has significant positive impacts on employee performance. In addition to training and selecting the appropriate communication model, companies should also work to improve the soft skills of their employees in order to improve their communication skills, but also to organize inter-department meetings to enhance communication between employees (Hee et al., 2019).

When it comes to logistics, then emphasis should be certainly placed on communication within the two highest-cost generating subsystems, warehouse and transport (Muhammad et al., 2014). Increasing transparency of information as well as improving communication in these sectors can result in significant cost savings. In transport, the existence of information and communication technology (ICT) is of particular importance. Such technology includes software for planning, routing, information transfer, complaint management and vehicle tracking. All these activities can be monitored using the transport management system (TMS). On the other hand, adequate information about goods which should be stored such as pack size, weight, volume, type of packing, etc. can significantly speed up and facilitate this process, resulting in additional savings and increased efficiency. All these activities can be facilitated by the implementation of a warehouse management system (WMS) (Muhammad et al., 2014). The application of these systems in transport and warehouse can have an effect on reducing errors in the performance of these processes.

Based on all previously said, the following hypotheses (Figure 1) were developed, which were then tested based on a survey conducted in the logistics sector of Serbia:

- H1a: The sources of stress vary depending on the primary business activity of the company;

- H1b: The sources of stress vary depending on the size of the company;

- H1c: The sources of stress vary depending on the position of the employee;

- H2a: Stress management varies depending on the primary business activity of the company;

- H2b: Stress management varies depending on the size of the company;

- H2c: Stress management varies depending on the position of the employee;

- H3a: The frequency of communication problems varies depending on the size of the company;

- H3b: The frequency of communication problems varies depending on the position of the employee. 


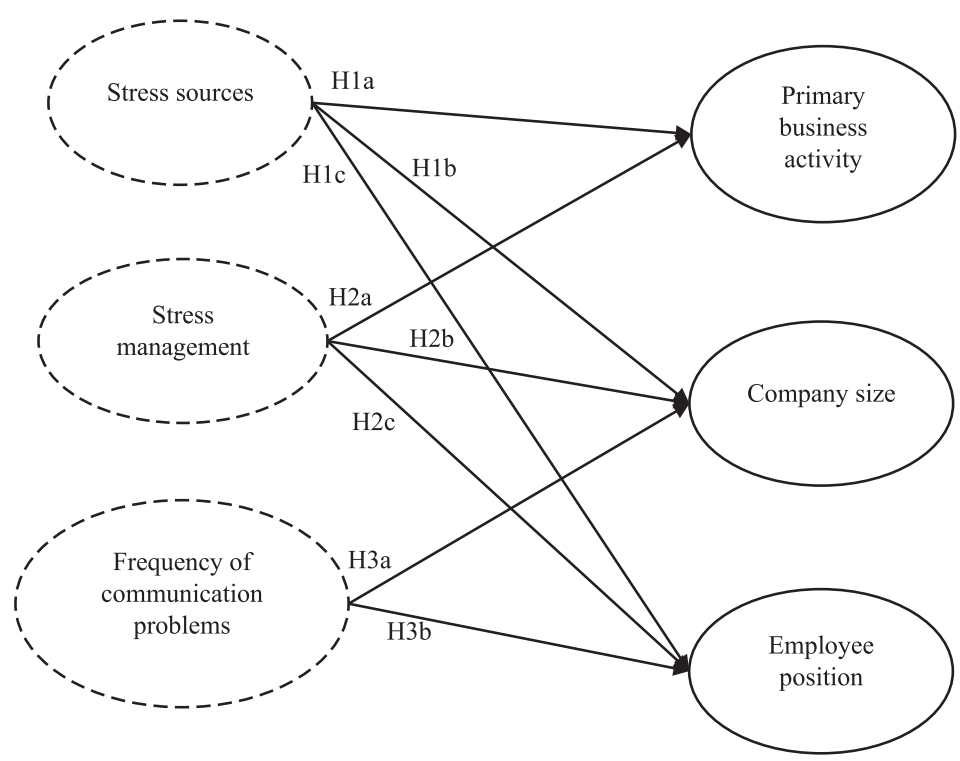

Fig. 1.

Model of Stress Sources, Stress Management and Frequency of Communication Problems

\section{Methodology}

Based on a systematic literature review, an was defined. As already mentioned, the three instrument for measuring the impact of stress and communication on the quality of the working environment in logistics companies basic dimensions identified in this paper are: sources of stress, stress management and frequency of communication problems (Table 2).

Table 2

Variable Description

\begin{tabular}{|c|c|c|}
\hline Dimension & Attributes & References \\
\hline Stress sources & $\begin{array}{c}\text { What are the most common stress } \\
\text { sources }\end{array}$ & Bhui et al., 2016 \\
\hline Stress management & How do you manage stress at work & $\begin{array}{c}\text { Antony Joe Raja and Vijayakumar, } \\
2017\end{array}$ \\
\hline $\begin{array}{c}\text { Frequency of communication } \\
\text { problems }\end{array}$ & $\begin{array}{c}\text { How frequent are communication } \\
\text { problems }\end{array}$ & Hola, 2012 \\
\hline
\end{tabular}

Based on the developed instrument, an appropriate questionnaire was created. The questionnaire was used as a data collection tool. The questionnaire was composed of two parts, where the first part of the questionnaire included questions to determine the characteristics of the respondents and the companies in which they work, while the questions in the second part were asked in order to determine the impact 
of stress and communication on the quality of the working environment. In order to process the results, a 5-point Likert scale was used. As mentioned earlier, the questionnaire was intended for logisticians in Serbia. An electronic version of the questionnaire was sent to 150 addresses.
The survey was conducted in the period from January to February 2020. The questionnaire was sent to 150 addresses but returned from 123. After selective analysis, responses that were not completely filled were excluded from further analysis which ended with 101 fully filled questionnaires.

\section{Table 3}

Descriptive Statistics

\begin{tabular}{|c|c|}
\hline Sample & Share $(\%)$ \\
\hline \multicolumn{2}{|l|}{ Primary business activity } \\
\hline International logistics and freight forwarding & 37.62 \\
\hline Retail & 22.77 \\
\hline Contract logistics $-3 \mathrm{PL}$ & 20.79 \\
\hline Distribution and domestic transport & 18.81 \\
\hline \multicolumn{2}{|l|}{ Company size } \\
\hline Very small company (up to 10 employees) & 35.64 \\
\hline Small company (up to 50 employees) & 19.80 \\
\hline Medium company (50-300 employees) & 19.80 \\
\hline Large company (>300 employees) & 24.75 \\
\hline \multicolumn{2}{|l|}{ Position } \\
\hline Logistics Assistant & 21.78 \\
\hline Procurement Referent & 22.77 \\
\hline Supply Chain Specialist & 34.65 \\
\hline Logistics Manager & 20.79 \\
\hline
\end{tabular}

A sample of 101 responses was then statistically processed. Based on the results and Table 3 , it can be concluded that respondents work in companies of different sizes, in different positions and in different sectors. Namely, the results of Table 3 show that the largest number of respondents work in companies dealing with international logistics and freight forwarding $(37,62 \%)$, followed by retail companies $(22,77 \%)$, contract logistics 3PL (20,79\%) and distribution and domestic transport $(18,81 \%)$. In terms of company size, the largest number of respondents wirk in very small companies $(35,64 \%)$, large companies $(24,75 \%)$ and small and medium-sized companies (19,80 each). When observing position of the employee, then it can be concluded that the largest number of respondents is employed in the position of supply chain specialist $(34,65 \%)$, followed by the procurement referent $(22,77 \%)$ and logistics assistant $(21,78 \%)$, while the smallest percentage of respondents are employed as logistics managers $(20,79 \%)$.

In order to confirm the reliability of the analyzed sample, IBM SPSS 25 software was used. The reliability test showed that all Cronbach's $\alpha$ were greater than 0.749 , indicating acceptable level of internal consistency between items. Given that the reliability of the sample was confirmed, hypotheses were then tested. The results of the testing are presented below. 


\section{Results}

Based on the hypotheses developed in chapter 2 , this section analyzes the results of their testing in more details. Testing was performed using linear regression (Swanson, 2016; Helmy et al., 2018). The results of the linear regression are shown in Table 4. In this paper three dependent and three independent variables were used. Primary business activity, company size and position are dependent variables, while independent are stress sources, stress management and frequency of communication problems.

Table 4

Linear Regression Results

\begin{tabular}{|l|c|c|c|c|c|c|}
\hline \multirow{2}{*}{\multicolumn{2}{|c}{ Variables }} & Primary business activity & \multicolumn{2}{c|}{ Company size } & \multicolumn{3}{c|}{ Position } \\
\cline { 2 - 8 } & Beta & Sig. & Beta & Sig. & Beta & Sig. \\
\hline Stress sources & 0,400 & 0,000 & 0,239 & 0,016 & 0,580 & 0,000 \\
\hline Stress management & 0,491 & 0,000 & 0,400 & 0,000 & 0,273 & 0,006 \\
\hline Frequency of communication problems & $/$ & $/$ & 0,602 & 0,000 & 0,231 & 0,020 \\
\hline
\end{tabular}

Based on the results (Table 4) and the value of $\beta=0,400, p<0,001$, it can be concluded that the sources of stress vary depending on the primary business activity of the company which is in accordance with Bhui et al. 2016. The results of the conducted research also indicate that there is a difference since employees working in international logistics and freight forwarding and trade companies have identified short time deadlines as a major source of stress. Employees in companies dealing with contract logistics - 3PL cite pressure from superiors as a major source of stress. Disagreement with colleagues stands out as the main source of stress for employees in distribution and domestic transport companies. These results can provide a good basis for companies to define corrective measures to eliminate sources of stress. On the basis of all previously said, it can be concluded that hypothesis $\mathrm{H} 1 \mathrm{a}$ is supported.

According to the results (Table 4) and the value of $\beta=0,239, p<0,05$, it can be concluded that the sources of stress vary depending on the size of the company. The analysis of the results of the conducted research shows that the most common source of stress in very small companies is short time deadlines. In small companies, the most common source of stress is insufficient knowledge/experience to do a specific job. Pressure from superiors stands out as the most significant source of stress in mid-sized companies. In large companies, disagreement with colleagues is a major source of stress. Based on these data, it can be concluded that large companies should work to improve internal communication, while smaller companies should work to improve the competencies and knowledge of their employees. Bearing previously said in mind, it can be said that the $\mathrm{H} 1 \mathrm{~b}$ hypothesis is supported.

Based on the values in Table $4(\beta=0,580, p$ $<0,001)$, it can be concluded that the sources of stress vary depending on the position of the employee, which is consistent with Bhui et al. 2016. The results of the survey show that employees in the position of 
logistics assistant cite short time deadlines as the main source of stress. Employees in the position of procurement referent cite insufficient knowledge/experience to perform a particular job as a major source of stress. Pressure from superiors stands out as the main source of stress for employees in the position of supply chain specialist. For employees in the position of the logistics manager, the main sources of stress are disagreement with colleagues and pressure from superiors. With these results in mind, companies would be able to define training according to the employee's position in order to learn how to cope with stress. This way, hypothesis $\mathrm{H} 1 \mathrm{c}$ is also supported.

According to the results (Table 4) and the value of $\beta=0,491, p<0,001$, it can be said there is a difference in stress management depending on the primary business activity of the company, which is in accordance with a previous study (Antony Joe Raja and Vijayakumar, 2017). Survey results show that employees of international logistics and freight forwarding companies, as the most effective method for stress management, cite seeking help from their superiors. Employees of trade and contract logistics - 3PL companies, as the most effective method for stress management, identify problem-solving with the help of a colleague. Problem-solving at a joint meeting has proven to be the most effective method for employees of distribution and domestic transport companies. These results provide an excellent basis for companies in defining stress management strategies at work. On the basis of all previously said, it can be concluded that hypothesis $\mathrm{H} 2 \mathrm{a}$ is supported.

Based on the values in Table $4(\beta=0,400, p$ $<0,001)$, it can be concluded that there are differences in stress management depending on the size of the company. Namely, the results of the survey show that in very small companies, seeking help from superiors is the most applicable stress management method. In small companies, problem-solving with the help of a colleague is the most effective method. Problem-solving at a joint meeting stands out as the most effective method in medium and large companies. This way, hypothesis $\mathrm{H} 2 \mathrm{~b}$ is also supported. In this regard, companies can compare their own stress management system with the above. In certain situations, other forms of stress management that are not specific to the certain segment to which company belongs may be applied.

According to the results (Table 4) and the value of $\beta=0,273, p<0,05$, there was a difference in stress management depending on the position of the employee. Based on the results of the survey, it can be concluded that employees in the position of logistics assistant identified seeking help from superiors as the most effective method for stress management. In addition to this method, employees in the position of procurement referent also identified problem-solving with the help of colleagues. Problem-solving at a joint meeting stands out as the most effective method for employees in the position of supply chain specialist. For employees in the position of the logistics manager, problem-solving with the help of colleagues is the most effective method for stress management. Based on these results, the importance of communication can be concluded, which is why companies should conduct this type of training for all positions. On the basis of all previously said, it can be concluded that hypothesis $\mathrm{H} 2 \mathrm{c}$ is supported.

The results (Table $4, \beta=0,602$, $p<$ $0,001)$ confirmed that the frequency of 
communication problems depends on the size of the company, which is consistent with (Hola, 2012). Namely, the results of the conducted research show that in very small companies communication problems occur on a daily basis. In small companies, communication problems occur 3-4 times per week. Communication problems in medium-sized companies occur once every 3 weeks, while in large companies they occur once a month. These results show that communication problems are more pronounced in smaller companies where employees are less likely to have received training in communication. For this reason, these companies should work in order to improve the communication and soft skills of their employees. Bearing previously said in mind, it can be said that the $\mathrm{H} 3 \mathrm{a}$ hypothesis is supported.

Based on the results (Table 4) and the value of $\beta=0,231, p<0,05$, it can be said that there is a difference in the frequency of communication problems depending on the position of the employee. Namely, the results of the conducted research show that employees in the position of logistics assistant believe that problems arise on a daily basis. Employees in the position of procurement referent estimate that communication problems occur 3-4 times per week, while employees in the position of supply chain specialist estimate that communication problems occur once every 3 weeks. Communication problems that occur once a month are rated as the most frequent by employees in the position of logistics manager. It can be concluded that the most frequent communication problems are at the lower hierarchical levels. For this reason, companies should organize mentors for employees at lower positions, as a form of training, but also as intermediaries in communicating with hierarchically higher positions. This way, hypothesis $\mathrm{H} 3 \mathrm{~b}$ is also supported.

\section{Conclusion}

The quality of the working environment can be a key factor when choosing a particular company to work for. Stress and communication are the main quality indicators of the working environment. The degree of exposure to stress, in addition to the characteristics of the job itself, depends on the person itself but also on the stress management implemented by the company. For this reason, it is very important for the company to find and implement the right stress management strategy since the consequences of stress affect not only employees but also the company. These consequences are most often reflected in reduced efficiency, productivity, increased costs, etc. In addition to choosing an appropriate stress management strategy, companies should also work on developing their employees' soft skills as well as organizing training for employees to learn how to cope with stress at work. Besides stress, communication is one of the most common indicators of the quality of the working environment. Just like stress, communication can have a significant impact on the company, since communication can have both internal and external consequences. For this reason, these two indicators have been used in order to assess their impact on the quality of the working environment in the logistics sector of Serbia.

A questionnaire was formed for the purpose of the survey, which was sent to 150 addresses, of which 101 answers were analyzed after excluding those answers 
that were not complete. The hypothesis development aimed to determine whether there were differences in stress sources, stress management and frequency of communication problems depending on the primary business activity of the company, company size and position of the employee. All hypotheses were supported. After testing the hypotheses, it was concluded that the stress sources vary depending on the primary business activity of the company. When observing company size, based on the results, it can be concluded that short time deadlines are the most common source of stress in very small companies, while disagreement with colleagues stands out as the most significant source in larger companies. It can be concluded that stress management methods vary depending on the primary business activity of the company, where employees in international logistics and freight forwarding companies identified seeking help from superiors as the most effective method. In companies dealing with trade and contract logistics - 3PL, the most effective method of stress management is to solve problems with the help of colleagues. Problemsolving at a joint meeting has proven to be the most effective method for employees in companies dealing with distribution and domestic transport. The results of hypothesis testing show that there is a difference in the frequency of communication problems, both depending on the size of the company and depending on the position of the employee. Based on the analysis of the results, it can be concluded that communication problems are most common in very small companies, as well as with employees in the position of logistics assistant. The results obtained are a good basis for people in practice for defining corrective actions but also for further analysis of this issue. An analysis of other indicators of the quality of the working environment, as well as their impact on employees and on the company itself, stands out as a direction for future research. In addition, it would be good to carry out similar or identical research in other markets so that the results obtained can be compared.

\section{Acknowledgements}

This paper was supported by the Ministry of Education, Science and Technological Development of the Republic of Serbia, through the project TR 36006.

\section{References}

Adu-Oppong, A.; Agyin-Birikorang, E. 2014. Communication in the workplace: Guidelines for improving effectiveness, Global Journal of Commerce \& Management Perspective 3(5): 208-213.

Antony Joe Raja, V.; Vijayakumar, V. 2017. A study on Stress Management in Various Sectors in India, International Journal of Management 8(1): 50-61.

Bhui, K.; Dinos, S.; Galant-Miecynikowska, de Jongh, B.; Stansfeld, S. 2016. Perceptions of work stress causes and effective interventions in employees working in public, private and non-governmental organisations: a qualitative study, BJPsych Bulletin 40(6): 318-325.

Colligan, T.W.; Higgins, E.M. 2006. Workplace stress: Etiology and consequences, Journal of Workplace Behavioral Health 21: 89-97.

Cooper, C.L.; Marshall, J. 1976. Occupational sources of stress: A review of the literature relating to coronary heart disease and mental ill health, Group and Organization Studies 13: 11-28.

Hee, O.C.; Qin, D.A.H.; Kowang, T.O.; Husin, M.M.; Ping, L.L. 2019. Exploring the Impact of Communication on Employee Performance, International Journal of Recent Technology and Engineering 8(3S2): 654-658. 
Helmy, K.A.; ElMokadem, M.Y.; el Bary, A.A.; ElSayeh, M. 2018, The Impact of Logistics Performance on Competitive Advantage: The Case of Freight Transportation in Egypt, Journal of WEI Business and Economics 7(1): 33-47.

Hola, J. 2012. Internal communication in the small and medium sized enterprises, Ekonomika a Management 15(3): 32-45.

Ismail, A.; Saudin, N.; Ismail, Y.; Abu Samah, A.J.; Abu Bakar, R.; Aminudin, N.N. 2015. Effect of Workplace Stress on Job Performance, Economic Review: Journal of Economics and Business 13(1): 45-57.

Jacobs, M.A.; Yu, W.; Chavez, R. 2015. The effect of internal communication and employee satisfaction on supply chain integration, International Journal of Production Economics 171: 60-70.

Kilibarda, M.; Pajić, V.; Andrejić, M. 2019. Human resources in logistics and supply chains: current state and trends. In Proceedings of the $4^{\text {th }}$ Logistics International Conference, 142-151.

Ližbetinova, L. 2015. Quality evaluation of internal communication in logistics companies, Logi, České Budějovice: Institute of Technology and Business in České Budějovice, No 1: 80-89.
Muhammad, M.; Saahar-Saabar, S.; Hasan, H.; Mohd Fiah, A.F.; Mohd Nor, A. 2014. Effective Communication Systems for Malaysian Logistics Industry, ProcediaSocial and Behaviour Sciences 130: 204-215.

Sadlowska-Wrzesinska, J.; Moscicka-Teske, A. 2016. Relations between stress potential of work features and occupational commitment of transport workers in the context of optimization of logistics strategy of a company, IFAC-PapersOnLine 49(12): 1761-1766.

Swanson, D. 2016. Transportation price benchmarking: implications for firm performance, Benchmarking: An International Journal 23(4): 1015-1026.

Thomas, R.W.; Esper, T.L.; Stank, T.P. 2011. Coping with time pressure in interfirm supply chain relationships, Industrial Marketing Management 40: 414423.

Yaslioglu, M.; Ozgur Karagulle, A.; Baran M. 2013. An Empirical Research on the Relationships between Job Insecurity, Job Related Stress and Job Satisfaction in Logistics Industry, Procedia - Social and Behavioral Sciences 99: 332-338. 